\title{
American Mineralogist thanks the 2020 reviewers
}

American Mineralogist greatly values the time and effort of the 2020 reviewers, especially in such an unprecedented year. The Journal thrives due to the commitment of many people. It could not do so without the support of these crucial volunteers.

\section{REVIEWERS}

Abdurixit, Azmat
Agangi, Andrea
Agee, Carl B.
Allen, Charlotte
Alvaro, Matteo
Amador, Elena
Anderson, Alyssa
Andreozzi, Giovanni B.
Andrews, Benjamin J.
Anenburg, Michael
Appold, Martin
Arletti, Rossella
Armbruster, Thomas
Arndt, Nicholas T.
Artacho, Emilio
Arzilli, Fabio
Ashley, Kyle T.
Asimow, Paul D.
Ayers, John C.
Ayuso, Robert
Azadbakht, Zeinab
Büttner, Steffan
Bacik, Peter
Bakker, Ronald
Balan, Etienne
Baldermann, A.
Baldwin, Julia A.
Balić-Žunić, Tonči
Ballhaus, Chris
Ballouard, Christophe
Barbeau, David
Baronnet, Alain
Bassett, William A.
Basu, Abhisek
Becker, Udo
Beirau, Tobias
Belmonte, Donato
Belokoneva, E.L.
Bendaoud, Abderrahmane
Benigni, Pierre
Bernardini, Simone
Berryman, Eleanor J.
Biagioni, Cristian
Bilenker, Laura D.
Bindi, Luca
Bismayer, Ulrich

\begin{tabular}{|c|c|}
\hline Bloch, Elias & Cerantola, Valerio \\
\hline Blundy, Jon D. & Cernuschi, Federico \\
\hline Bodnar, Robert J. & Cerri, Guido \\
\hline Boffa Ballaran, Tiziana & Chan, Jerry \\
\hline Bolfan-Casanova, Nathalie & Chariton, Stella \\
\hline Bonal, Lydie & Chayka, Ivan \\
\hline Bonnet, Guillaume & Che, Xu Dong \\
\hline Bosi, Ferdinando & Chen, Bin \\
\hline Boudreau, A.E. & Cheng, Hao \\
\hline Bouhifd, Ali & Cheng, Lilu \\
\hline Boulard, Eglantine & Christy, Andrew G. \\
\hline Boulliard, Jean-Claude & Chukanov, Nikita V. \\
\hline Boyce, Jeremy & Chumakov, Alexander \\
\hline Bradbury, Harold & Cicconi, Maria Rita \\
\hline Breiter, Karel & Ciobanu, Cristiana L. \\
\hline Brenan, James & Cisneros Lazaro, Deyanira \\
\hline Brenna, Marco & Clark, Alisha \\
\hline Briggs, Sophie & Cloos, Mark \\
\hline Brillo, Jürgen & Cloutis, Edward A. \\
\hline Bristow, Thomas & Codeço, Marta S. \\
\hline Broekmans, Maarten A. & Colás, Vanessa \\
\hline Broom-Fendley, Sam & Collins, William \\
\hline Brounce, Maryjo & Colombo, Fernando \\
\hline Brown Jr., Gordon E. & Cook, Nigel \\
\hline Brueckner, Stefanie & Cooper, Mark A. \\
\hline Brzozowski, Matthew & Cordier, Patrick \\
\hline Buatier, Martine & Cornell, David \\
\hline Bucholz, Claire & Costin, Gelu \\
\hline Burgess, Katherine & Cottrell, Elizabeth \\
\hline Burnley, Pamela C. & Courtney-Davies, Liam \\
\hline Burns, Peter C. & Coustel, Romain \\
\hline Bussweiler, Yannick & Craw, David \\
\hline Bystricky, Misha & Crichton, Wilson \\
\hline Cabri, Louis J. & Cruz-Uribe, Alicia M. \\
\hline Callegaro, Sara & Cuadros, Javier \\
\hline Camara, Fernando & Cui, Huan \\
\hline Cametti, Georgia & Cynn, Hyunchae \\
\hline Caraballo Monge, Manuel A. & De Ligny, Dominique \\
\hline Carmichael, Sarah & De Villiers, Johan \\
\hline Carpenter, Michael A. & Debret, Baptiste \\
\hline Carrez, Philippe & Della Ventura, Giancarlo \\
\hline Cartigny, Pierre & Depmeier, Wulf \\
\hline Case, Bruce & Dewaele, Agnès \\
\hline Castroviejo, Ricardo & Ding, Shuo \\
\hline Cavazos-Álvarez, Jaime A. & Dobrzhinetskaya, Larissa \\
\hline Cawood, Peter & Dorais, Michael J. \\
\hline Cempírek, Jan & Dorfman, Susannah M. \\
\hline Cenki-Tok, Benedicte & Dos Santos, Antonio M. \\
\hline
\end{tabular}

Dragovic, Besim

Du, Jianguo

Dubacq, Benoît

Duffy, Thomas S.

Durand, Cyril

Dushyantha, Nimila

Dyar, Melinda D.

Dyck, Brendan

Echigo, Takuya

Effenberger, Herta

Ehm, Lars

Elongo, Vanessa

Encarnacion, John

Erdmann, Saskia

Ertl, Andreas

Ewing, Rodney C.

Fabio, Bellatreccia

Faccenda, Manuele

Fan, Dawei

Fan, Hongrui

Ferrando, Simona

Ferraris, Giovanni

Finnis, Mike

Fitros, Michalis G.

Flemming, Roberta L.

Forster, Michael

Fougerouse, Denis

Fournelle, John H.

Frank-Kamenetskaya, Olga V.

Fregola, Rosa Anna

Fries, Marc

Fritsch, Emmanuel

Fritz, Jörg

Frost, B.R.

Frost, Carol D.

$\mathrm{Fu}$, Bin

Fuchs, Yves

Fukushi, Keisuke

Gadd, Michael G.

Gaft, Michael

Gaillard, Fabrice

Gaillot, Anne-Claire

Galvez, Matthieu

Ganguly, Jiba

Garofalo, Paolo

Gartman, Amy

Garvie, Laurence A.

Gatta, G. Diego 
Gavrilenko, Maxim

Gavryushkin, Pavel N.

Geiger, Charles A.

Gemmi, Mauro

Ghosh, Biswajit

Ghosh, Sujoy

Giebel, Robert J.

Giester, Gerald

Gin, Stephane

Gleadow, Andrew J.

Gleason, Arianna

Goldmann, Simon

Gomi, Hitoshi

González-García, Diego

Gosh, Dipta

Grangeon, Sylvain

Gregory, Dan

Gregory, Daniel D.

Grew, Edward S.

Grey, Ian E.

Grieco, Giovanni

Groppo, Chiara

Guedes, Sandro

Hadi, Jebril

Hagen-Peter, Graham

Haggerty, Stephen

Halenius, Ulf

Hanchar, John

Hanley, Jacob

Harald, Behrens

Harlaux, Matthieu

Haroldson, Erik L.

Harries, Dennis

Harris, Chris

Hatert, Frederic

Hausladen, Debra

Hazen, Robert M.

$\mathrm{He}$, Hongping

Heaney, Peter J.

Hedenquist, Jeff

Heinrich, Chris

Hejny, Clivia

Henry, Darrell J.

Hermann, Joerg

Hervig, Richard

Hetherington, Callum

Hilairet, Nadege

Hiraga, Takehiko

Hofmeister, Anne M.

Holder, Robert

Hopp, Jens

Hsieh, Wen-Pin

Hsu, Han

Hu, Jinping

$\mathrm{Hu}$, Qingyang

$\mathrm{Hu}$, Siyu

Huber, Chris

Hughes, John M.

Hui, Hejiu

Hummer, Daniel R.

Huraiová, Monika

Iacovino, Kayla

Ibanez-Mejia, Mauricio

Iitaka, Toshiaki
Ingrin, Jannick

Iveson, Alex

Jacobsen, Steven D.

Jaret, Steven

Jenkins, David M.

Jercinovic, Michael J.

Jiang, Shao-Yong

Jiang, Zhaoxia

Jing, Zhicheng

Joachim-Mrosko, Bastian

Johnson, Tim

Jollands, Michael

Jones, Rhian H.

Kamada, Seiji

Kamenetsky, Vadim S.

Kaminsky, Felix

Kampf, Anthony R.

Kanzaki, Masami

Kapsiotis, Argyrios

Karel, Breiter

$\mathrm{Ke}$, Shan

Kearns, Stuart L.

Keller, Duncan S.

Kent, Adam J.

Keppler, Hans

Ketcham, Richard A.

King, Penelope L.

Kissin, Stephen A.

Kleeberg, Reinhard

Klemetti, Erik W.

Klemme, Stephan

Ko, Byeongkwan

Koch-Müller, Monika

Koděra, Peter

Koeberl, Christian

Kojitani, Hiroshi

Kolitsch, Uwe

Komabayashi, Tetsuya

Komatsu, Kazuki

Krivovichev, Sergey V.

Kroeker, Scott C.

Kroonenberg, Salomon

Krot, Alexander N.

Kruhl, Jörn

Kubicki, James

Kunz, Martin

Kusiak, Monika A.

Kwon, Kideok D.

Kyono, Atsushi

Lai, Xiaojing

Lamadrid, Hector

Lamb, William M.

Lane, Melissa D.

Lanson, Bruno

Large, Ross

Larsen, Rune

Łasocha, Wiesław

Lassin, Arnault

Le Roux, Véronique

Lee, Seungyeol

Legros, Hélène

Leinenweber, Kurt

Lenaz, Davide

Lepore, Giovanni O.
Leung, Kevin

Li, Baosheng

Li, Wei

Li, Xiao-Chun

Li, Xinyang

Li, Yuan

Li, Yunguo

Liang, Hua-Ying

Lin, Jung-Fu S.

Ling, Mingxing

Litasov, Konstantin D.

Liu, Jiachao

Liu, Xi

Liu, Yan

Lo Presti, Leonardo

Locock, Andrew

Louvel, Marion

Lowry, Anthony

Lu, Anhuai

Lu, Zunli

Lumpkin, Gregory R.

Luvizotto, George

Lykova, Inna

Müller, Axel

Müntener, Othmar

$\mathrm{Ma}, \mathrm{Chi}$

MacRae, Colin

Magnall, Joseph

Majzlan, Juraj

Makhluf, Adam

Makovicky, Emil

Malaspina, Nadia

Mallik, Ananya

Mancktelow, Neil

Maner, James L.

Manning, Craig E.

Manthilake, Geeth

Mao, Yajing

Mao, Zhu

Marquardt, Katharina

Marshall, Edward

Martin, Andrew

Martucci, Annalisa

Maslennikov, Valeriy

Mathur, Ryan

Matthews, Simon

Matzen, Andrew K.

Mavrogenes, John A.

McCaig, Andrew

McCarthy, Anders

McCloy, John S.

McCollom, Thomas

McCubbin, Francis

McDonald, Andrew M.

McKeown, David A.

McNulty, Brian

Mercurio, Mariano

Merlini, Marco

Metcalf, Rodney

Mihailova, Boriana

Miller, Calvin F.

Mills, Stuart J.

Milodowski, Antoni

Miozzi, Francesca
Miranda, Ana

Mitchell, Roger

Mittlefehldt, David

Miyajima, Nobuyoshi

Mohammadi, Nadia

Molina, José F.

Molinari, Simone

Montouillout, Valérie

Moore, Lowell R.

Moore, Nicole

Morin, Guillaume

Morrison, Shaunna

Mueller, Thomas

Myhill, Bob

Nabelek, Peter

Nabyl, Zineb

Nakajima, Yoichi

Nathwani, Chetan

Nazzareni, Sabrina

Neave, David A.

Nestola, Fabrizio

Newcombe, Megan E.

Nishihara, Yu

Nowak, Marcus

O’Neill, Hugh St.C.

Oberti, Roberta

Okazaki, Keishi

Olds, Travis A.

Orlandini, Omero

Ovsyannikov, Sergey V.

Pack, Andreas

Padrón-Navarta, José Alberto

Paktunc, Dogan

Palin, Richard

Palke, Aaron C.

Palmer, Martin

Pamato, Martha

Pamukcu, Ayla

Panczer, Gerard

Paonita, Antonio

Papineau, Dominic

Paris, Michael

Parker, Steve

Parsons, Ian

Pasero, Marco

Pearson, Graham D.

Pereira, Inês

Peterman, Emily M.

Petitgirard, Sylvain

Petrik, Igor

Pettke, Thomas

Phillips, Brian L.

Piña García, Rubén

Pichavant, Michel

Pingitore, Nicholas

Pinti, Daniele

Pisch, Alex

Plasil, Jakub

Plotinskaya, Olga Y.

Pokrovski, Gleb S.

Pommier, Anne

Post, Jeffrey E.

Pring, Allan

Pullen, Alex 
Putirka, Keith D.

Putnis, Andrew

Rakovan, John F.

Rampe, Elizabeth

Reddy, Steven M.

Redhammer, Guenther J.

Reed, Mark

Rickard, David

Rioux, Matthew

Rogers, Deanne

Roggensack, Kurt

Rollion-Bard, Claire

Rosiere, Carlos Alberto

Ross, Nancy L.

Rossman, George R.

Rottier, Bertrand

Rubin, Alan E.

Ruf, Alexander

Ruiz-Agudo, Encarnacion

Runyon, Simone

Ryan, Jeffrey G.

Ryan-Davis, Juliet

Sánchez-Navas, Antonio

Saini, Naurang

Sakamaki, Tatsuya

Sanloup, Chrystèle

Sato, Tomoko

Satta, Niccolò

Saunders, James

Saylor, Joel

Schönleber, Andreas

Schindler, Michael

Schmieder, Martin

Scott, James

Seitz, Sisanne

Semprich, Julia

Sharp, Zachary D.

Sharygin, Victor V.

Shaw, Cliff S.

Shea, Joshua

Shea, Thomas

Shen, Andy H.

Shen, Bing

Sherman, David

Shibazaki, Yuki

Shieh, Sean R.

Shinozaki, Ayako

Shu, Qihai
Simon, Adam C.

Singer, David M.

Singerling, Sheryl A.

Sirbescu, Mona-Liza C.

Sircombe, Keith

Skála, Roman

Skogby, Henrik

Slaby, Ewa

Smit, Karen

Smith, Evan

Smith, Evan M.

Smith, Ian E.

Smith, Martin

Smythe, Duane J.

Solomatova, Natalia V.

Song, Shuguang

Song, Wen Lei

Sossi, Paolo

Spear, Frank S.

Spencer, Christopher

Spiekermann, Georg

Stackhouse, Stephen

Stagno, Vincenzo

Stebbins, Jonathan F.

Steele-MacInnis, Matthew

Stepanov, Aleksandr S.

Stowell, Harold H.

Streck, Martin J.

$\mathrm{Su}$, Benxun

Sugiura, Yuki

Sun, Keke

Tabacchi, Gloria

Tacker, Christopher

Tagami, Takahiro

Tagirov, Boris

Tailby, Nicholas

Takir, Driss

Tao, Renbiao

Tappe, Sebastian

Taylor, Sandra

Teng, H. Henry

Tennakoon, Sumudu

Terasaki, Hidenori

Thatcher, Diana

Thiry, Yves

Thomas, Jay

Thomas, Rainer

Thompson, Aaron
Thompson, Elizabeth C.

Thompson, Samuel

Tielke, Jacob

Tkalcec, Beverley

Tollan, Peter

Tollefsen, Elin

Tomascak, Paul

Tomkins, Andrew

Tornos, Fernando A.

Townsend, Joshua

Trail, Dustin

Trautmann, Christina

Tribaudino, Mario

Tschauner, Oliver

Tsikos, Harilaos

Tsujino, Noriyoshi

Uhlik, Peter

Ulrich, Thomas

Umemoto, Koichiro

Van Driessche, Alexander

Veksler, Ilya V.

Vennari, Cara E.

Vezin, Herve

Vezinet, Adrien

Vignola, Pietro

Viviano, Christina

Von Rudorff, Guido

Vuilleumier, Rodolphe

Waight, Tod

Wakabayashi, John

Wallace, Adam F.

Walowski, Kristina

Walton, Anthony W.

Walton, Erin

Wang, Christina Y.

Wang, Da

Wang, Kuo-Lung

Wang, Ping

Wang, Rui

Wang, Xiaolei

Wang, Xiaolin

Wang, Yejian

Watson, Bruce B.

Webb, Samuel M.

Weerasooriya, Rohan

Wei, Chen

Weisberg, Michael

Welch, Mark D.
Werts, Kevin

Wheeler, John

White, Lee

White, Noel

Whittaker, Michael

Wilding, Martin

Williams, Lynda B.

Winkler, Bjoern

Withers, Anthony

Wojtulek, Piotr Marian

Woodland, Alan B.

Wu, Fuyuan

Wu, Xiang

Wu, Zhongqing

Xia, Xiao-Ping

Xiao, Yuanyuan

Xie, Yuling

Xie, Zhanjun

Xiong, Xiaolin

$\mathrm{Xu}$, Cheng

Xu, Haijun

$\mathrm{Xu}$, Xisheng

Yakymchuk, Chris

Yang, Hexiong

Yang, Tao

Yang, Xiaozhi

Yang, Zhen

Yang, Zhiming

$\mathrm{Ye}, \mathrm{Yu}$

Yoder, Claude H.

Yuan, Shunda

Yuan, Xueyin

Zakharov, David

Zega, Tom

ZHAI, Shuangmeng

Zhang, Dongzhou

Zhang, Fuxiang

Zhang, Hongfu

Zhang, Jin S.

Zhang, Lejun

Zheng, Jianping

Zhong, Xin

Zhou, Jiaxi

Zhou, Mei-Fu

Zhu, Mengqiang

Ziberna, Luca

Zolensky, Michael E.

Zviagina, Bella B. 